\title{
Minimal Invasive Plate Osteosynthesis (MIPO) Technique Using Anterolateral Approach for Treating Closed Proximal Humerus Fracture
}

\author{
Ismail HD, Ph.D, Boedijono DR*, MD, Hidayat H, MD, Simbardjo DS, MD \\ Department of Orthopaedic \& Traumatology, Faculty of Medicine University of Indonesia-Cipto \\ Mangunkusumo Hospital, Jakarta, Indonesia \\ *Department of Orthopaedic \& Traumatology Fatmawati General Hospital, Jakarta, Indonesia
}

\begin{abstract}
Proximal humerus fracture is the second most common fracture of the upper extremity and presents several unique problems such as anatomical complexity, high risk of avascular necrosis, minimal bone stock for purchase, significant morbidity, and lack of a universally accepted treatment. Recent treatments for proximal humerus fractures include use of minimally invasive plate osteosynthysis (MIPO). The aim of this cross-sectional study was to evaluate the outcomes of our less invasive technique using a modified anterolateral approach for treatment of proximal humerus fractures. Ten such operative procedures were performed in patients of varying age and with varied mechanism of injury from 2002-2011. All cases were conducted in an acute setting. There were no cases of infection and the functional outcome scores were good. This approach represents an alternative treatment for closed proximal humerus fracture but more extensive studies are needed.
\end{abstract}

Key Words:

Proximal humerus fracture, MIPO, less invasive, anterolateral approach

\section{INTRODUCTION}

Fracture of the proximal humerus is the second most common fracture of the upper extremity following distal forearm fractures ${ }^{1}$. In people older than age 65 -years-old, proximal humerus fracture is the third most common fracture, after hip fracture and Colles' fracture ${ }^{1}$. Proximal humerus fracture present several unique problems, which must be considered in order to achieve the best treatment results. First, the proximal humerus has a complex anatomy. The rotator cuff is a critical functional structure that must be reconstructed following proximal humerus fracture ${ }^{2}$. Second, the proximal humerus is vascularised by the anterior circumflex artery and arcuate artery, which are both prone to injuries, thereby increasing the risk of avascular necrosis.
The ascending branch of the anterior circumflex humeral artery (artery of Liang) has been demonstrated by Gerber to provide most of the blood flow to the articular segment ${ }^{3}$. Third, in proximal humerus fractures, there is minimal bone stock to purchase. Regional differences in the proximal humerus must be taken into account when attempting to reduce tuberosity fragments. For instance, the cortex of the proximal humerus near the greater tuberosity becomes progressively thicker as it proceeds distally. In fractures of the thinnest cortical bone, the fracture lines can be difficult to appose. Fourth, proximal humerus fracture is associated with significant morbidity, leading to functional impairment lasting at least 3 months ${ }^{4}$. Displaced proximal humerus fractures generally result in long-term functional disability ${ }^{5}$. This type of injury is usually sustained after a moderateenergy fall in individuals with low bone density ${ }^{4}$. To date, there is no consensus on the optimal treatment of complex fractures of the proximal humerus.

Treatment type is primarily determined by examining the radiographs and computed tomographic (CT) scans of the proximal humerus and then classifying the injury according to the Neer classification. An anteroposterior (AP) view of the shoulder in the plane of the scapula, a lateral view of the scapula (Y view), and a supine axillary view are necessary to initially assess a proximal humerus fracture. If the degree of displacement of the humeral head or tuberosity fragments is uncertain, an axial CT study with 2-mm sections is obtained ${ }^{6}$.

Management of displaced proximal humerus fractures has evolved toward humeral head preservation. Treatment should be guided by careful assessment of vascular status, bone quality, fracture pattern, and degree of comminution, as well as patient factors, such as age and activity level. Patients who are medically unstable or inactive are poor candidates for surgery and instead may be treated with sling immobilization until the fracture heals. The ultimate goal is maximum shoulder function and minimal shoulder pain ${ }^{5}$. Recently, the advances in treatment for proximal humerus fracture have involved minimally invasive plating 
osteosynthesis (MIPO), which offer minimal soft tissue damage and rapid and improved healing of the fracture ${ }^{7,8}$. The surgical approaches commonly used for MIPO in proximal humerus fractures are the transdeltoid lateral approach, anterolateral approach, and deltopectoral approach 9 . The aims of study were to evaluate the outcomes of our less invasive plating technique using a modified anterolateral approach in proximal humerus fracture treatment.

\section{MATERIALS AND METHODS}

We treated 12 patients with 12 complex fractures of the proximal humerus from 2002-2011 using the less invasive plating osteosynthesis technique with an anterolateral approach. Based on criteria reported by Tingart et al., for all cases in this study, preoperative bone quality was judged to be good with strong trabeculae in the cephalic segment and a thickness of the cortex of the proximal diaphysis of more than $3.5 \mathrm{~mm}^{10}$. For follow-up, two patients were not available for contact, leaving total of ten fractures sustained by five men and five women with a mean age of 45.8-yearsold (range, 17 to $70 y$ ). (Table I). One patient had the implant removed in another country several months after operation.

Surgical treatment is chosen for patients with at least threepart fractures. There were seven cases of three-part fractures. Most of the three-part fractures involve fractures at the surgical neck and greater tuberosity (Figures 1a and 2a). The three cases of four-part fractures are the classic type involving fractures at the surgical neck, greater tuberosity and lesser tuberosity. All fractures were treated with reduction and internal fixation using the less invasive technique via a modified anterolateral approach. In eight patients, implants were a locally manufactured cloverleaf plate. A T-plate was used in one patient, and a proximal humerus plate was used in another patient. Bone graft was utilised in one case. Importantly, we did not use the 'true' MIPO, as we still opened the fracture site but minimised the skin and soft tissue incision. This is described as the modified anterolateral approach.

Our modified anterolateral approach involves several procedures as described herein. The patient is in supine position with sandbag placement behind the shoulder. An incision is made anteriorly from the usual lateral approach (anterolateral). After dissection of the subcutaneous tissue, blunt dissection of the deltoid is performed being careful to avoid distal extension in the area of the axillary nerve. After visualising the humerus, the rasp is used to prepare the distal portion of the bone for the plate. The fracture was reduced without further opening the fracture site. Reduction was evaluated by palpation at the fracture site and by observing humeral alignment. After reduction was achieved, the plate was slid from the proximal to distal position. Axillary nerve injury can be avoided by performing tunnelling under the axillary nerve and close to the bone. When inserting the plate, the axillary nerve bundle was retracted using an army navy retractor. Due to the anterolateral incision, injury of the axillary nerve bundle was avoided because the bundle was more prominent and more attached to lateral humerus. More small incisions were necessary for the placement of the distal screw (Figures 3a-c). Suction drainage was applied for three days in addition to administration of intravenous antibiotics. Postoperative management was dependent upon on the degree of operative stability achieved. Generally, pendulum exercises were started immediately and performed twice daily. A sling was worn for three weeks.

This was a cross-sectional study undertaken to evaluate outcomes of patients treated using this technique. Outcome criteria included review of bony union, range of motion (ROM) for flexion and abduction, and function. Telephone interviews were conducted and the latest radiographs were reviewed. As for functional outcome, patient review was conducted using the Disabilities of the Arms, Shoulder and Hand Score (DASH) score ${ }^{11}$, since most of the patients were lived in various regions across the country. The duration of follow up was between 4 and 65 months.

\section{RESULTS}

All fractures healed without delayed, malunion or non-union of any segments (Table II). The operative goal of reducing the fracture was met in all cases. We used bone grafting in one patient (case nine) (graft was composed of demineralized bone matrix). There were no cases of avascular necrosis of the humeral head. Three patients underwent a second operation for implant removal.

DASH scores ranged from $0-19.16$ (average, 9.56 + SD 6.06) and were evaluated at $3-74$ months post operatively (mean, 22.78 months). Three patients had DASH scores under 5, five patients scored 5-15 and the remainder had DASH scored higher than 15 (Figure 4). Based on the type of implant used in the surgery, regular plates showed better functional outcome than the cloverleaf plate (average DASH Score, 5.06 vs. 10.68). We also scored the functional results of this operation by measuring the range of motion (ROM) (flexion and abduction). Average range in flexion was $121.5^{\circ}$ + SD $22.2^{\circ}$ with two patients scoring $90-105^{\circ}$, five patients scoring $106-120^{\circ}$, and three patients scoring more than $120^{\circ}$ (Figure 5). Use of regular plates resulted in better range of flexion than the cloverleaf plate (average ROM $155^{\circ}+\mathrm{SD}$ $21.21^{\circ}$ vs. $\left.113.12+\mathrm{SD} 13.08^{\circ}\right)$. As for abduction, the average ROM was $95.5^{\circ}+\mathrm{SD} 23.8^{\circ}$; four patients scored 80 $89^{\circ}$ and six patients scored more than 900 . Use of regular plates also showed better range of abduction than the cloverleaf plate (average ROM, $130^{\circ}+\mathrm{SD} 42.43^{\circ}$ vs. 86.87 + SD $\left.7.04^{\circ}\right)$ (Table III).

There were no instances of wound infection or osteomyelitis. A demineralized bone graft was used in one patient. This 
Table I: Fracture type and treatment

\begin{tabular}{|llclll|}
\hline Case & Gender & Age (years) & \multicolumn{1}{c}{ Type of fracture } & Side & Type of treatment \\
\hline 1 & Male & 57 & 3-part, greater tuberosity & Left & Cloverleaf Plate \\
2 & Female & 60 & 4-part, greater \& lesser tuberosity & Right & Cloverleaf Plate \\
3 & Male & 31 & 3-part, greater tuberosity & Right & Cloverleaf Plate \\
4 & Female & 17 & 3-part, greater tuberosity & Right & Cloverleaf Plate \\
5 & Male & 30 & 3-part & Left & Cloverleaf Plate \\
6 & Female & 60 & 3-part, greater tuberosity & Right & T-Plate \\
7 & Female & 55 & 3-part, greater tuberosity & Right & Cloverleaf Plate \\
8 & Female & 70 & 4-part, greater \& lesser tuberosity & Right & Cloverleaf Plate \\
9 & Male & 54 & 4-part & Right & Cloverleaf Plate \\
10 & Male & 24 & 3-part & Left & Proximal Humerus Plate \\
\hline
\end{tabular}

Table II: Treatment Outcomes

\begin{tabular}{|lccccccc|}
\hline Case & Infection & Malunion & $\begin{array}{c}\text { DASH Score } \\
\text { (Time score taken } \\
\text { postoperatively) }\end{array}$ & 2nd Surgery & Pain & Flexion $\left(^{\circ}\right)$ & Abduction $\left(^{\circ}\right)$ \\
\hline 1 & No & No & $11.66(7$ months) & No & No & 110 & 90 \\
2 & No & No & $19.16(14$ months) & No & No & 90 & 80 \\
3 & No & No & $2.50(6$ months) & No & No & 110 & 85 \\
4 & No & No & $17.59(4$ months) & No & No & 120 & 90 \\
5 & No & No & $8.62(8$ months) & No & No & 115 & 80 \\
6 & No & No & $10.11(24$ months) & Plate Removal & No & 120 & 100 \\
7 & No & No & $11.12(74$ months) & No & No & 135 & 80 \\
8 & No & No & $4.62(65$ months) & Plate Removal & No & 105 & 100 \\
9 & No & No & $10.19(3$ months) & No & No & 170 & 160 \\
10 & No & No & $0(6$ months) & Plate Removal & & & 160 \\
\hline
\end{tabular}

Table III: Functional outcomes and ROM comparison between regular plate and cloverleaf plate

\begin{tabular}{|lccc|}
\hline No. & Parameter & Regular Plate & Cloverleaf Plate \\
\hline 1 & DASH Score, Average & $5.06 \pm 7.15$ & $10.68 \pm 5.72$ \\
2 & Flexion & $155^{\circ} \pm 21.21^{\circ}$ & $113.12^{\circ} \pm 13.08^{\circ}$ \\
3 & Abduction & $130^{\circ} \pm 42.43^{\circ}$ & $86.87^{\circ} \pm 7.04^{\circ}$ \\
\hline
\end{tabular}
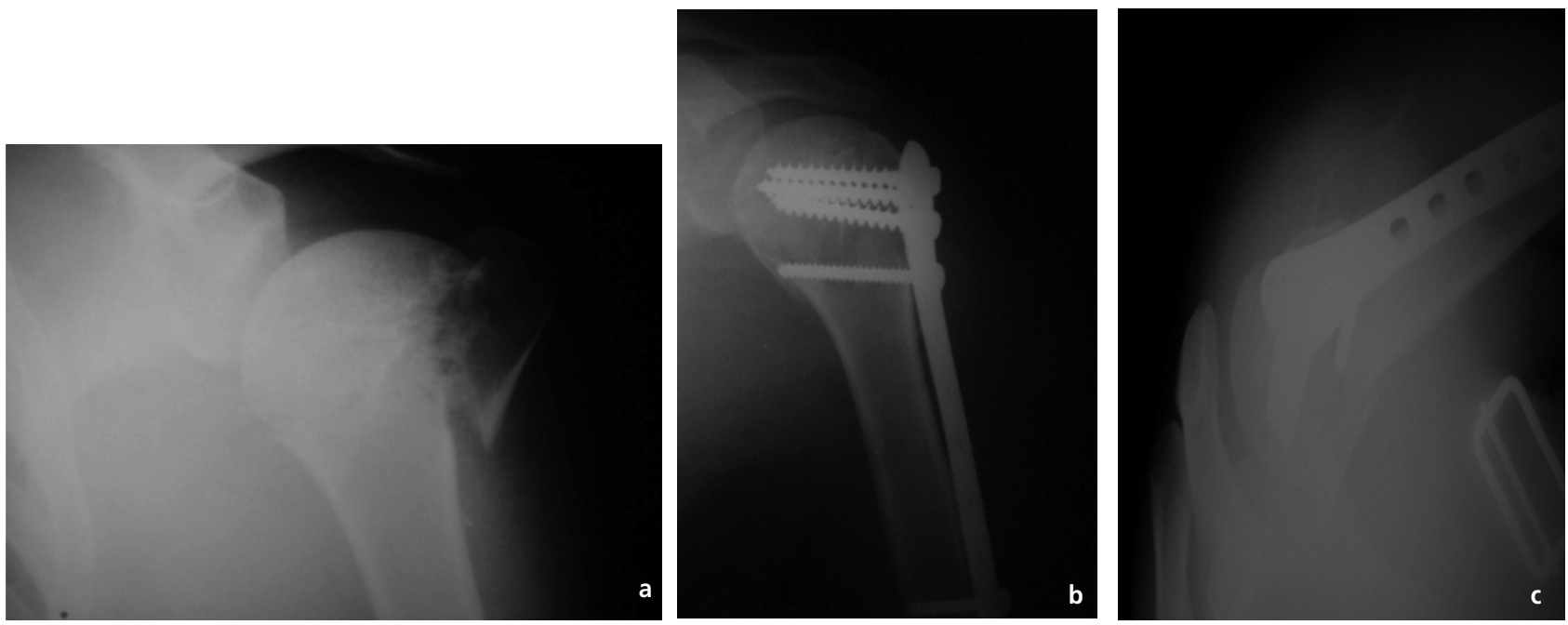

Fig. 1: 24-year-old male with Neer 3-part fracture, presentation in 2010; preoperative radiograph (Panel a) and postoperative radiographs (Panels b and c). 

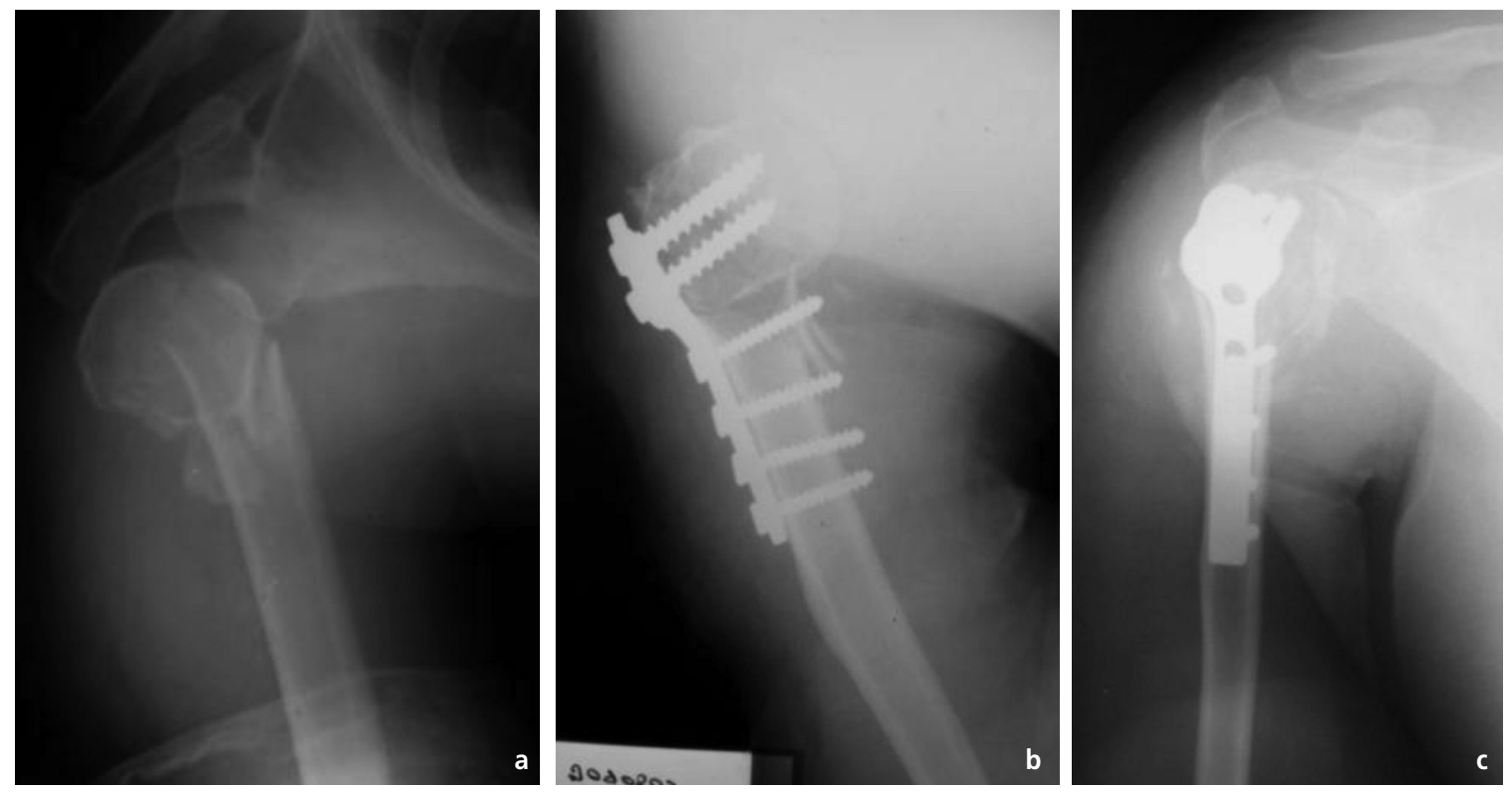

Fig. 2: Female, 65-year-old with Neer 3-part fracture, presented in 2003; preoperative radiograph (Panel a), postoperative radiographs (Panel b) and 2 years after the operation (Panel c).
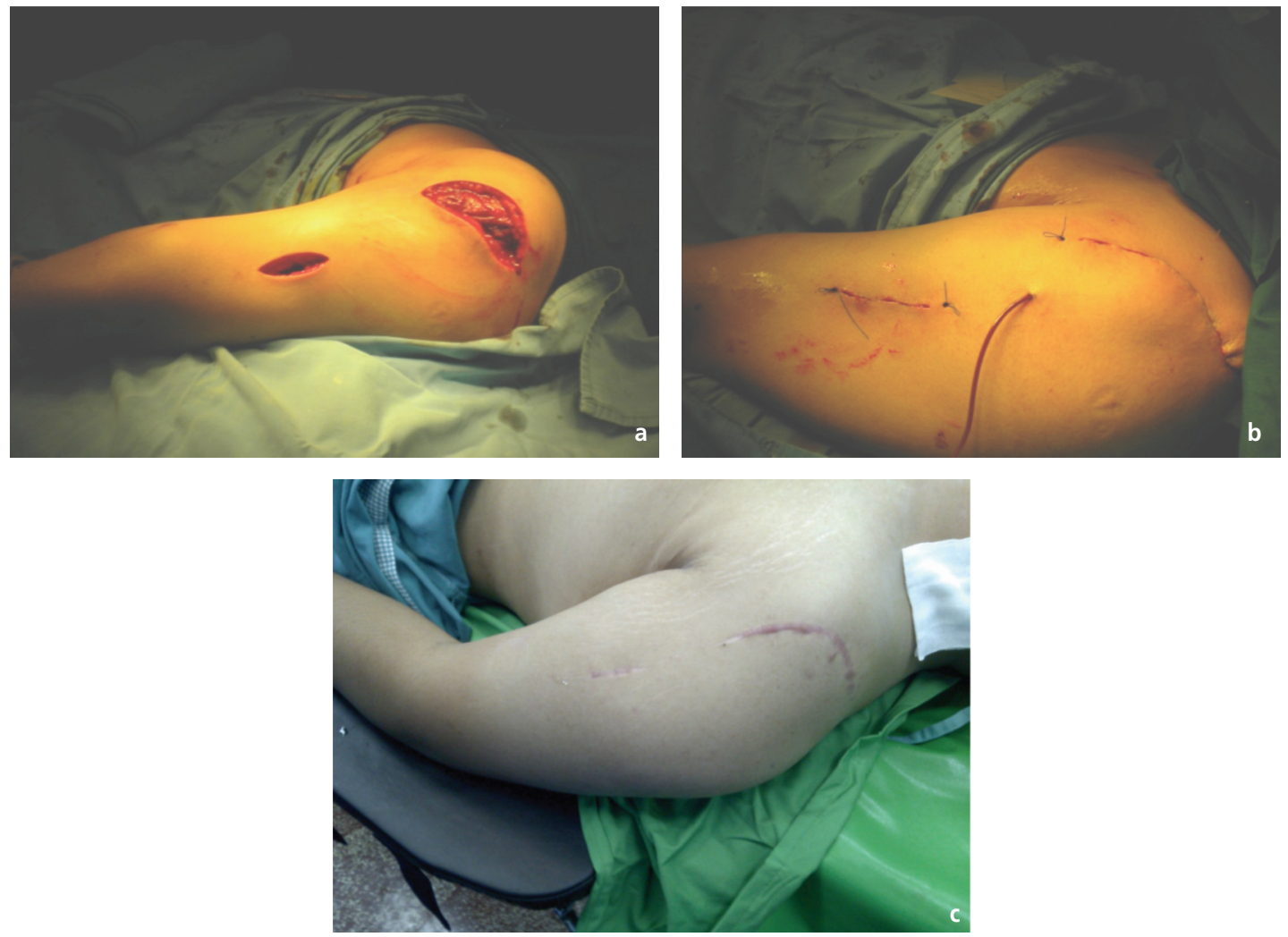

Fig. 3: Incisions made in anterolateral approach during operation (Panel a), postoperatively (Panel b) and during follow-up (Panel c). 


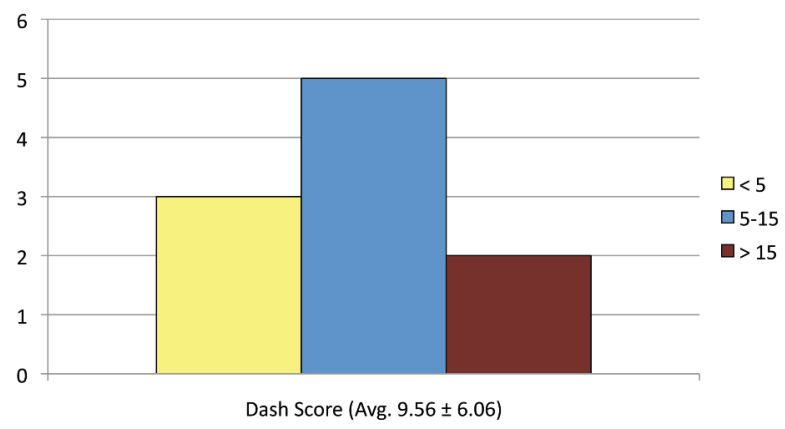

Fig. 4: Functional outcome as measured by DASH Score

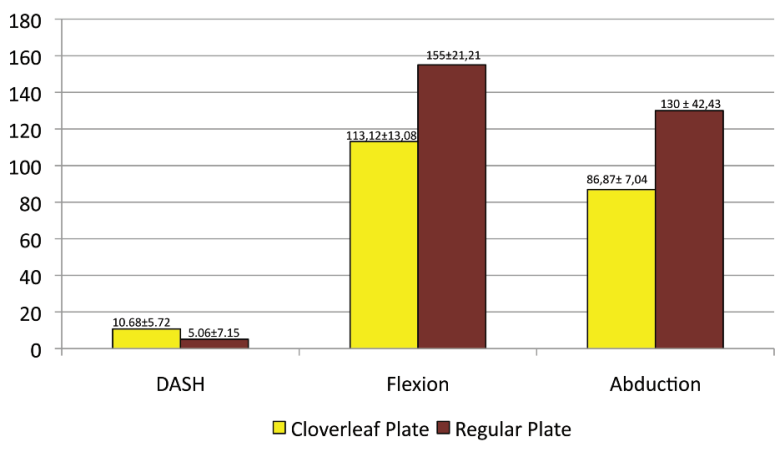

Fig. 5: Comparison of functional outcome and ROM (flexion and abduction) for the implants used in surgery.
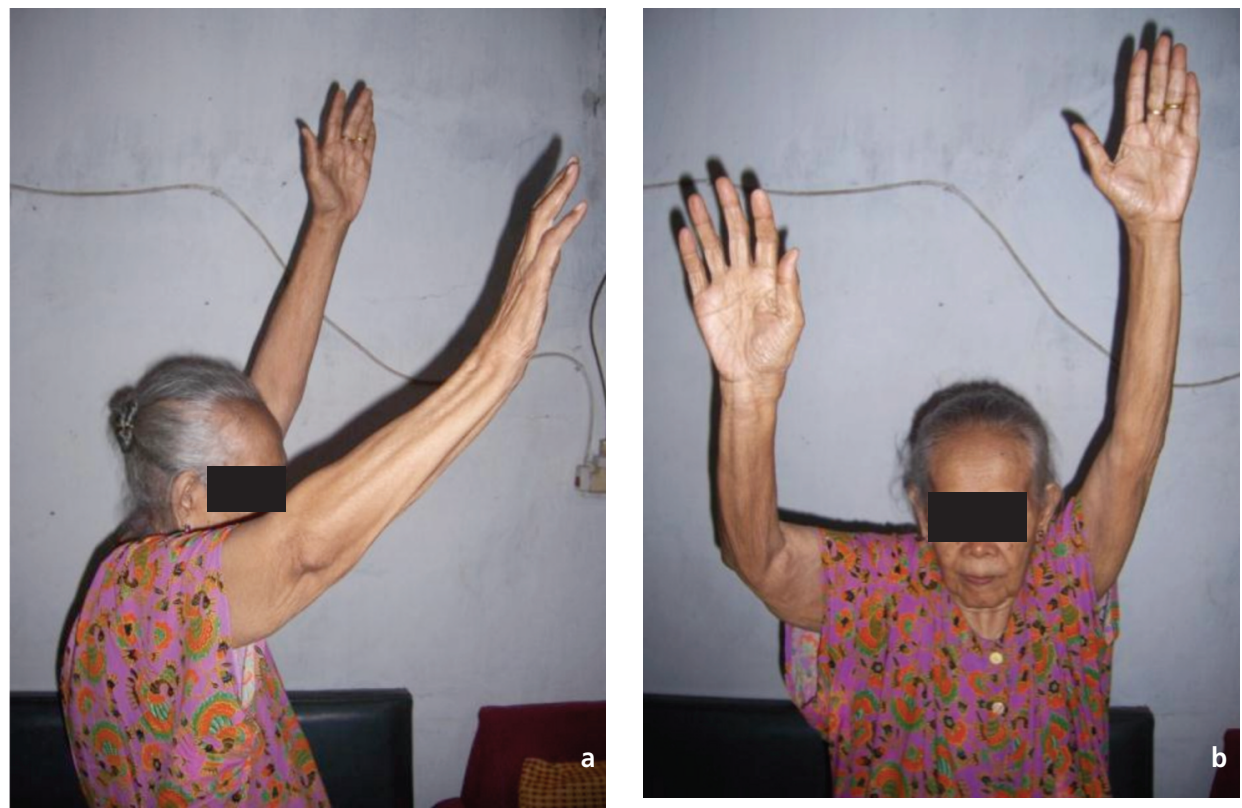

Fig. $6 a$ \& 6 b: Sixty-five months after removal of the implant, the patient has good function.

action was based on the surgeon judgement since the fracture was extensive. Interestingly, we encountered difficulty in forward flexion overall; in those three patients who had the implant removal, forward flexion was improved. The timing of clinical union was not known, as most patients did not come regularly to our clinic. Most patients were able to resume work 2-4 weeks postoperatively.

\section{DISCUSSION}

There is no consensus as to the optimal treatment of complex fractures of the proximal humerus. These fractures can be defined by a variety of classification systems including assessment of bone quality and metaphyseal comminution; all of these systems are prone to error. None gives a clear prognosis and direction for treatment. The difficulty in accurately classifying the fracture also creates problems in reporting outcomes. Overall, open reduction and internal fixation have yielded satisfactory results. The best results are obtained if the fractures are well reduced and reduction is maintained until healing has occurred. The goal must therefore be to select only those fractures that can be reduced for open reduction and internal fixation. Outcomes are dependent on various factors such as the type of fracture, the quality of the bone, the technique of reduction and fixation and the experience and skill of the surgeon. 
In the present series, 7 of 10 patients were younger than 60 years-old, and with a mean age of $45.8 \mathrm{y}+18,38 \mathrm{y}$; this is rather young for the types of fracture considered. In addition, five of the patients were men, who overall have better bone quality. Most other series have also included a much greater proportion of female patients ${ }^{12-15}$. Bone quality is an important factor in determining the outcome of open reduction and stabilisation of such fractures and should be considered when selecting treatment for a fracture of the proximal humerus. However, this was not a controlled study and we do not have evidence that fractures with poorer bone quality had poorer outcomes when treated in wit this method. The average DASH score of our patients at $9.56+$ SD 6.06 compared favourably to another recent study by Altman et al in 2011, who found that the average DASH score of proximal humerus fracture patients treated by MIPO averaged $25.95^{12}$. These numbers indicates a promising functional outcome. Sun et al also found that MIPO techniques combined with use of locking proximal humerus plates (LPHP) provided a satisfactory and effective method of treatment of proximal humerus osteoporotic fracture ${ }^{16}$.

A cloverleaf plate is commonly used in our country due to its easy availability and its reasonable price. In this study, we found that regular plate has better functional result. There are some studies investigating outcomes after the treatment of proximal humerus fractures using the locking plate. Most report good functional outcomes but only include a small number of subjects ${ }^{17,18,19}$. A multicentre prospective observational study conducted by Sudkamp et al. in 2010 concludes that the locking proximal humerus plate results in good functional outcomes in elderly patients with osteoporosis, but correct surgical techniques is important to avoid iatrogenic-related complications ${ }^{13}$.

The use of locking plates in MIPO is becoming a trend following the satisfactory results reported in several studies 7,8. This combination offers a good option for the treatment of proximal humeral shaft fractures and results in better functional outcomes and shorter hospital stays. It also has comparable rates of nonunion and lower rates of radial neuropathy compared to open procedures
Previously, a transdeltoid approach was often used in MIPO technique for proximal humerus fractures ${ }^{14}$. However, in the present series, we used the less invasive osteosynthesis technique via a modified anterolateral approach. This approach minimises the soft tissue damage, allows for improved and more rapid bone healing, less infection, less postoperative pain, and promotes early recovery. It is important to note, however, that this technique is technically demanding. It requires the orthopaedic surgical expertise since the surgical exposure and fracture reduction is limited. Another drawback of this technique is potential implant impingement, resulting in limited forward flexion sometimes requiring a secondary operation to remove the implant. To solve this problem, we suggest use of the low profile anatomical proximal humerus plate.

The aftercare of the patients in our series varied according to patient needs, because our aim was to have good functional outcomes in addition to anatomical healing. In all cases, we encountered difficulty in forward flexion; improvement occurred in the three patients who had the implant removed. This was probably due to the impingement that was caused by the implants (Figure 6a-b). We therefore suggest that as soon as the fracture is healed, the implant should be removed; alternatively, we suggest use of the low-profile anatomical proximal humerus plate at the first operation.

Further studies are warranted with increased numbers of treated patients and more use of the low-profile anatomical proximal plate to further evaluate outcomes in such cases.

\section{CONCLUSION}

The results showed that an anterolateral approach for treatment of proximal humerus fractures using the less invasive osteosynthesis technique can be expected to produce good treatment outcomes. It can be considered as an alternative option to the conventional open reduction and internal fixation technique. Results show relatively good DASH outcome scores and good patient satisfaction after the operation. 


\section{REFERENCES}

1. Baron JA, Barrett JA, Karagas MR. The epidemiology of peripheral fractures. Bone. 1996; 18(3): 209-13.

2. Fjalestad T, Hole MO, Blücher J, Hovden IA, Stiris MG, Strømsøe K. Rotator cuff tears in proximal humeral fractures: an MRI cohort study in 76 patients. Arch Orthop Trauma Surg. 2010; 130(5): 575-81.

3. Gerber C, Schneeberger AG, Vinh TS. The arterial vascularization of the humeral head: An anatomical study. J Bone Joint Surg [AM] 1990; 72: 1486-94.

4. Lee SH, Dargent-Molina P, Breart G, EPIDOS Group. Epidemiologie de l'Osteoporose Study. Risk factors for fractures of the proximal humerus: Results from the EPIDOS prospective study. J Bone Miner Res. 2002; 17: 817-25.

5. Nho SJ, Brophy RH, Barker JU, Cornell CN, MacGillivray JD. Innovations in the management of displaced proximal humerus fracture. J Am Acad Orthop Surg. 2007; 15:12-26.

6. Naranja RJ, Iannotti JP. Displaced three- and four-part proximal humerus fractures: Evaluation and management. J Am Acad Orthop Surg. 2000; 8: 373-82.

7. Rancan M, Dietrich M, Lamdark T, Can U, Platz A. Minimal invasive long PHILOS®-plate osteosynthesis in metadiaphyseal fractures of the proximal humerus. Injury (2010) Volume: 41, Issue: 12, Publisher: Elsevier Ltd, Pages: 1277-83.

8. Brunner A, Thormann S, Babst R. Minimally invasive percutaneous plating of proximal humeral shaft fractures with the Proximal Humerus Internal Locking System (PHILOS). J Shoulder Elbow Surg. 2011; 25:

9. Wong M, Bavonratanavech S. Humerus, proximal in AO manual of fracture management: Minimally invasive plate osteosynthesis (MIPO). City: Publisher; 2007.120-30

10. Tingart MJ, Apreleva M, von Stechow D, Zurakowski D, Warner JJ. The cortical thickness of the proximal humeral diaphysis predicts bone mineral density of the proximal humerus. J Bone Joint Surg [Br]. 2003; 85: 611-17.

11. Solway S, Beaton DE, McConnell S, Bombardier C. The DASH Outcome Measure User's Manual, Second Edition. Toronto: Institute for Work \& Health; 2002.

12. Altman GT, Gallo RA, Molinero KG, Muffly MT, Mascarenhas L. Minimally Invasive Plate Osteosynthesis for Proximal Humerus Fractures: Functional Results of Treatment. Am J Orthop. 2011; 40(3): 40-7

13. Sudkamp N, Bayer J, Hepp P, Voigt C, Oestern H, Kaab M, et al. Open Reduction and Internal Fixation of Proximal Humeral Fractures with Use of the Locking Proximal Humerus Plate: Results of a Prospective, Multicenter, Observational Study. The $J$ Bone Joint Surg. 2009; 91: 1320-8.

14. Acklin YP, Jenni R, Walliser M, Sommer C. Minimal invasive PHILOS-plate osteosynthesis in proximal humeral fractures. Eur J Trauma Emerg Surg. 2009; 35: 35-9.

15. Lau TW, Leung F, Chan CF, Chow SP. Minimally invasive plate osteosynthesis in the treatment of proximal humeral fracture. International Orthopaedics (SICOT). 2007; 31: 657-64.

16. Sun LJ, Chen H, Guo XS. LPHP combined with MIPO techniques for treatment of proximal humeral fractures in elderly osteoporotic patients. Chinese journal of hand surgery. 2009; 25(1):

17. Koukakis A, Apostolou CD, Taneja T, Korres DS, Amini A. Fixation of proximal humerus fractures using the PHILOS plate: early experience. Clin Orthop Relat Res. 2006; 442: 115-20.

18. Fankhauser F, Boldin C, Schippinger G, Haunschmid C, Szyszkowitz R. A new locking plate for unstable fractures of the proximal humerus. Clin Orthop Relat Res. 2005; 430: 176-81.

19. Björkenheim JM, Pajarinen J, Savolainen V. Internal fixation of proximal humeral fractures with a locking compression plate: a retrospective evaluation of 72 patients followed for a minimum of 1 year. Acta Orthop Scand. 2004; 75: 741-5. 